\title{
X-ray imaging of transplanar liquid transport mechanisms in single layer textiles
}

\author{
${ }^{1}$ Gannian Zhang, ${ }^{2}$ Rachna Parwani, ${ }^{3}$ Corinne A. Stone, ${ }^{2 \star}$ Asa H. Barber, ${ }^{1}$ Lorenzo Botto \\ ${ }^{1}$ School of Engineering and Materials Science, Queen Mary University of London, E1 4NS, United Kingdom \\ ${ }^{2}$ School of Engineering, University of Portsmouth, Portsmouth PO1 3DJ, United Kingdom \\ ${ }^{3}$ Defence Science \& Technology Laboratory, Porton Down, Salisbury SP4 0JQ, United Kingdom \\ KEYWORDS (Transplanar liquid transport, single layer textiles, $X$-ray imaging)
}

\begin{abstract}
Understanding the penetration of liquids within textile fibers is critical for the development of nextgeneration smart textiles. Despite substantial research on liquid penetration in the plane of textile, little is known about how the liquid penetrates in the thickness direction. Here we report a time-resolved high-resolution X-ray measurement of the motion of the liquid-air interface within a single layer textile, as the liquid is transported across the textile thickness following the deposition of a droplet. The measurement of the time-dependent position of the liquid meniscus is made possible by the use of ultra-high viscosity liquids (dynamic viscosity from $10^{5}$ to $2.5 \cdot \times 10^{6}$ larger than that of water). This approach enables imaging due to the slow penetration kinetics. Imaging results suggest a three-stage penetration process with each stage being associated with one of the three types of capillary channels existing in the textile geometry, providing insights into the effect of the textile structure on the path of the three-dimensional liquid meniscus. One dimensional kinetics studies show that our data for the transverse penetration depth $\Delta x_{L}$ vs. time do not conform to a power law, and that the measured rate of penetration for long times is smaller than that predicted by Lucas-Washburn kinetics, challenging commonly held assumptions regarding the validity of power laws when applied to relatively thin textiles.
\end{abstract}

\section{INTRODUCTION}

The prediction of liquid transport in textiles plays an important role in many applications, from the design of liquid repellent textiles for skin protection ${ }^{1,2}$, to the development of resin impregnation methodologies ${ }^{3,4}$, and the manufacturing of textiles with improved wearability ${ }^{5,6}$. Classical problems in this field are the prediction of wicking rates in experiments where the textiles are put in contact with large liquid volumes ${ }^{7}$, the analysis of the liquid distribution due to moisture condensation ${ }^{8}$, and the prediction of in-plane and transplanar liquid transport following drop deposition ${ }^{9,10}$. In this paper we focus on the last problem.

The process of liquid wicking in a textile when a drop is deposited on its surface can be conceptually described as composed of two stages, namely transplanar penetration (also called transplanar uptake ${ }^{11}$ or transplanar wicking ${ }^{5,12}$ ) and in-plane spreading ${ }^{13}$. The first stage refers to liquid transport normal to the plane of the textile, and occurs temporally before in-plane spreading (although the two mechanisms can be simultaneous in the early stages of spreading). While substantial knowledge has been accumulated in understanding issues of in-plane transport $7,9,14,15,16,17,18,19$, 20 , investigations on the mechanisms of transplanar transport are scarce ${ }^{5,11,12}$. Transplanar penetration is a relatively fast process ${ }^{21}$, and is therefore often neglected when examining the long-time behavior of the liquid.

In the case of single layer textiles, spontaneous transplanar liquid penetration (i.e., with no imposed pressure on the liquid) has so far been limited to approximate measurements with the so-called "demand wettability" method ${ }^{11}$. In this method, the fabrics are placed horizontally directly in contact with either a large tank of liquid, or a thin paper fully saturated by liquid. The liquid uptake is recorded by measuring the weight loss of the liquid source with a microbalance. In refs. [11] and [12] the mass of absorbed liquid was reported to vary approximately linearly with time, and then saturate to a constant value. The demand wettability method does not provide data that can aid the analysis of microscopic transport mechanisms.

For multilayer textiles, Bencsik et al. ${ }^{22}$ employed Nuclear Magnetic Resonance (NMR) to visualize the oil distribution in a three-layer textile as a function of an applied compressive load. The volume of oil in the textile was analyzed as a function of the coordinate normal to the plane of the textile for different compressive loads. The three-dimensional distribution of the liquid within the textile was not considered. It should be emphasized that liquid penetration driven by a pressure 
gradient follows a different dynamic from the liquid penetration driven by a capillary pressure. Transplanar wicking in a two-layer knitted textile from a reservoir of liquid being continuously supplied to the textile via a capillary tube was studied by Birrfelder et al ${ }^{5}$ using $X$ ray radiography. Two-dimensional cross sections of the textile were presented showing the liquid front in both layers. A mechanism of liquid transport was proposed whereby intra-yarn pores were responsible for transplanar penetration of liquid within one layer while large inter-yarn pores were associated with layer-tolayer transport. However the paper did not present quantitative information on the motion of the liquid front. Transplanar wicking in a three-layer textile from a reservoir of liquid was studied using X-ray radiography by Rossi et al ${ }^{23}$. The water distribution in the textile was characterized by the attenuation intensity of the X-rays. The work focused on the amount of water being absorbed by individual layers. No description of microscopic transport was attempted. X-ray tomography was used by Weder et al ${ }^{24}$ to investigate transplanar wicking in a four-layer woven textile. Water distribution as a function of the thickness of the material was presented. This paper focused primarily on monitoring the amount of liquid in each layer over time rather than observing the liquid front movement. The spatial resolution was relatively coarse, with pixel sizes of about $70 \mu \mathrm{m}$.

Data for transplanar liquid penetration is available for relatively thin porous media other than textiles. Bayramli and Powell studied transplanar penetration in carbon fiber tows ${ }^{25}$. A carbon fiber tow is geometrically similar to a textile yarn, as it is formed by concentric layers of packed carbon fibers oriented along the tow axis. The liquid penetration depth normal to the fibers was measured by examining the weight of the wet tow, and found to be proportional to the square root of time, as expected from the Lucas-Washburn kinetics ${ }^{16}$. Data on transplanar liquid penetration across paper is available in the publication from Karppinen et al. ${ }^{26}$, where for the transplanar transport due to the deposition of a droplet was analyzed. The authors measured the intensity of diffuse light reflection from the paper. The light reflection curve showed a monotonic decrease with time and an asymptotic plateau. The penetration depth was not characterized and no attempt to model the data was made. A simulation of drop penetration in a paper layer of thickness larger than the drop diameter was carried out by Hyväluoma et al. ${ }^{27}$. The transplanar liquid penetration depth was found to be much smaller than the prediction by the Lucas-Washburn law. Although the structure of paper has some similarity to that of a textile, paper lacks the hierarchy of pore scales that characterizes the structure of textiles. In particular, paper is characterized by a marked scale separation between the size of the largest pores in the porous structure and the thickness of the paper layer. Such separation of scales is not ensured in textiles, which have relatively large pores.
The issue of scale separation also relates to the distinction between single and multilayer textiles. A sufficiently thick multilayer textile can be considered a bulk, three-dimensional porous medium sample. It is therefore expected that the long time capillary wicking behavior for liquid penetration in a thick textile follows continuum theories, in contrast for a single layer textile, the time taken for the fluid to cross the textile thickness may not be enough time for an asymptotic behavior to be established.

From the point of view of the development of theoretical models, there is large uncertainty regarding the three-dimensional distribution of liquid within porous layers when a drop is placed on them. The theoretical model of Patnaik et al ${ }^{17}$ for liquid penetration in a single layer textile assumes that transplanar penetration occurs uniformly under the droplet, and that the liquid front is planar. In contrast, the model of Starov et al. ${ }^{28}$, developed to analyze liquid penetration in millimetric glass and metal filters, assumes that the advancing liquid front inside the material is approximately hemispherical. In these studies the microscopic, three-dimensional distribution of the liquid is not analyzed, and a continuum approach is taken. The model of Gillespie for spreading in paper ${ }^{14}$ neglects the initial stage of transplanar penetration. The lack of experimental data that could aid the development of theoretical models is one of the main motivations for the current work.

Experimental challenges in measuring transport by $\mathrm{Nu}$ clear Magnetic Resonance, Neutron Radiography and X-ray Computed Tomography (XCT) include loss of signal over long experimental duration and insufficient spatial and temporal resolution. Measuring transplanar liquid transport is particularly challenging, as for low viscosity liquids the time scale of liquid penetration across the textile layer is small (the required time resolution for water is typically below $0.1 \mathrm{~s}^{21}$ )

In this paper, to overcome time resolution limitations, we use silicone fluids with ultra-high viscosities to slow down the penetration kinetics and enable time-resolved XCT imaging. In contrast to all previous work on single-layer textiles, and most work on multilayer textiles, we report the three-dimensional, microscopic distribution of the liquid within the textile, and discuss potential transport mechanisms that account for the multiscale structure of the textile.

\section{MATERIAL AND METHODS}

High viscosity silicone oils $(100 \%$ polydimethylsiloxane) were used to achieve direct imaging of the transplanar penetration. The viscosities are $\eta=97 \mathrm{~Pa} \cdot \mathrm{s}$ (Sigma Aldrich) and $\eta=2.4 \cdot 10^{3} \mathrm{~Pa} \cdot \mathrm{s}$ (Clearco Products). Both oils are non-volatile at room temperature. The oil-air interfacial tension is reported by the companies to be in the range of $19-21 \mathrm{mN} \cdot \mathrm{m}^{-1}$. PDMS displays shear thinning behavior at sufficiently large values of the shear rate ${ }^{29}$. In our case, because of the 
relatively small shear rate values characteristic of flow within the textile (we estimate $\gamma \dot{\sim} \frac{\gamma}{\mu \mathrm{d}} \cong 1-10 \mathrm{~s}^{-1}$ ), nonNewtonian effects are neglected in the analysis.

The plain woven textiles are produced by yarns in two orientations aligned at $90^{\circ}$ to one another. Fibers in the yarns are cotton and polyester mixed at a ratio of $19: 1^{3}$ (95\% cotton). The typical dimensions of the textile are: thickness $\mathrm{h} \sim 300 \mu \mathrm{m}$; inter-yarn pore size $150 \cdot 20 \mu \mathrm{m}^{2}$, inter-fiber pore size $d_{i} \sim 3 \mu \mathrm{m}$, fiber diameter $d \sim 10 \mu \mathrm{m}$.

For the sample preparation, textile is cut into square sections with approximate side length of $5 \mathrm{~mm}$ and mounted vertically on a standard XCT bin using adhesive. Due to the extremely high viscosity, small amount of both oils are manipulated with needles to form droplets and carefully transferred to the sample surface. The droplet height is on the same order of magnitude as the textile thickness.

XCT was carried out only with the highest viscosity liquid $\left(\eta=2.4 \cdot 10^{3} \mathrm{~Pa} \cdot \mathrm{s}\right)$ using an X-ray microscope (520 Versa, Carl Zeiss, USA), illustrated in Fig. 1. A $2.6 \mu \mathrm{m}$ isotropic voxel size was achieved from imaging samples using a total of 601 projections across 360 degrees of sample rotation. Each projection was collected using a $0.5 \mathrm{~s}$ exposure time. The obtained $2 \mathrm{D}$ projections were reconstructed to a $3 \mathrm{D}$ volume using a filtered back projection algorithm implemented in the manufacturer's software. A standard Shepp-Logan filter, Gaussian filter (strength of 0.5) and beam hardening correction (strength of 0.05 ) was applied to the projections. The imaging conditions allowed the shortest imaging time possible while maintaining a relatively high spatial resolution. The 70-minute experimental duration allowed the collection of three tomography scans.

Radiography images were obtained by X-ray beam transmission along the $y$ axis and through the sample to the detector as indicated in Fig. 1. The radiography projections offer a superimposed view of all the $\mathrm{xz}$ planes along the $y$ axis thus enable real-time monitoring of the liquid front within the textile. Pixel size, number of projections, exposure and waiting time between projections were set to $1.296 \mu \mathrm{m}, 1200,4 \mathrm{~s}$ and $1 \mathrm{~s}$ respectively.

The shift of the X-ray intensity profiles at successive times shows the displacement of the spatially averaged advancing liquid front in the textile in the $x$ direction. To compute the displacement, intensity threshold values $115,120,122,125$ and 126 were selected. For each threshold value, the displacement $\Delta x_{L}$ is calculated using the equation: $\Delta x_{L}=x_{t}-x_{0}$, where $x_{t}$ is the coordinate of the advancing liquid front at $t$ and $x_{0}$ is the initial coordinate of the liquid front at $t=0 \mathrm{~s}$.

The obtained XCT slices from the resulting three dimensional reconstruction is segmented into liquid and textile (including background) phases by machine learning-based segmentation (Weka, 3.9.0, ImageJ, U. S. National Institute of Health, Bethesda, Maryland, USA) to render the liquid distribution in the textile more evident. By coloring the liquid, air and textile phase red, green and purple respectively in the segmentation results, we are able to clearly visualize the time-evolved liquid distribution in the textile. In addition, when only the liquid phase needs to be presented, the liquid phase is shown in black while the textile and air phases are shown in white for a good contrast.

To classify image points as belonging to textile, air, or liquid we use a Weka learning scheme ${ }^{30}$, as implemented in ImageJ. Briefly, the Weka learning scheme is based on applying a series of filters, such as edge detectors (Laplacian and Sobel, Gabor etc.) texture filters (minimum, maximum, median, entropy etc.) and noise reduction filters (Gaussian, Kuwahara, Lipschitz etc.) to an original image. The filtered images form an "image pipeline", representing different image features. Pixel samples are manually labelled in the original image and subsequently the labels will be applied to the corresponding pixels from the images in the pipeline. The pixel values will then give a range for the liquid, air and textile phase which are then used as training sets to make a classifier. Applying the classifier to images to be segmented will classify the pixels automatically and then present the liquid, air and textile phase in different colors.

\section{RESULTS AND DISCUSSION}

Our sample is characterized by an ordered multiscale structure whose main geometric features are illustrated in Fig. 2. The inter-fiber pores are the smallest pores within the textile. The textile is formed by relatively thick (diameter $\sim 100 \mu \mathrm{m}$ ) yarns disposed in a mesh-like arrangement. Each yarn is composed of small diameter fibres (diameter $10 \mu \mathrm{m}$ ) twisted around the yarn's central axis. In the following text we will denote the relatively large (linear dimension $\sim 100 \mu \mathrm{m}$ ) pores of the textile as inter-yarn pores.

The shape of the center line of each yarn in the out-ofplane direction is sinusoidal. Each yarn first passes above a perpendicular row of yarns and then below the successive one. This arrangement yields a saddleshaped contact region, whose projection on the $y-z$ plane is indicated in Fig. 2 by a dark, dashed region. Because of the irregular surface of each yarn, the contact region has a finite maximum thickness of roughly $10 \mu \mathrm{m}$ (which in turn is of the order of the fiber size). The inter-fiber pores, i.e. the small interstices within the yarns, have a typical diameter of about $3 \mu \mathrm{m}$. This is slightly smaller than each fiber diameter. We have examined the diameters of the capillary pores and of the fibers in the XCT images by averaging over 15 fibers for each diameter value reported. 
Fig. 3 shows front (yz) and side (xz) views of the droplet outside of the textile. The droplet spreads laterally while simultaneously penetrating in the normal direction. The motion of the air-liquid-solid contact line is relatively slow, and is hindered by the large out-of-plane roughness of the textile (which results from the wavy shape of the yarns) as well as by the presence of fibers that "stick" out of plane, piercing the droplet. Out-ofplane fibers constitute unavoidable structural features that are due to the rupture of fiber bundles. This rupture is the result of the weaving process or the repeated manual handling of the textile sample. The front view in Fig. 3 shows that the shape of the droplet projected onto the yz plane is approximately circular for $\mathrm{t}=$ 25 mins. At later times the projection of the contact line onto the yz plane tends to assume a polygonal shape. This feature is likely to result from the triple line conforming to the regular microstructure of the textile, as suggested by studies of droplet spreading on microfabricated surfaces formed by regular arrays of pillars ${ }^{31}$.

Fig. 4 shows side $(x z)$ views of the liquid within the textile for $\eta=2.4 \cdot 10^{3} \mathrm{~Pa} \cdot \mathrm{s}$, focusing on the inter-yarn pore region. To render the distribution of the liquid more evident, Weka segmentation (see Material and Methods section) was used to associate different colors to the liquid, air and solid phases. For $t=25$ mins, the inter-yarn pore is filled with liquid for $3 / 4$ of its depth. For the remaining 45 minutes, the motion of the liquidair interface in the inter-yarn pore region is almost negligible. The dynamics for $t<25 \mathrm{~min}$ is not accessible due to the experimental requirement of 25 minutes to complete one tomography scan. If the largest pores were modeled as circular channels of uniform crosssectional radius $\mathrm{R}$ and depth $\mathrm{h}$, capillary penentration would require a time $T \cong \frac{2 h^{2}}{R} \frac{\eta}{\gamma \cos 9}$ of the order of few minutes. For example, $\mathrm{T} \cong 5$ mins assuming $\mathrm{h} \cong$ $250 \mu \mathrm{m}, \mathrm{R} \cong 50 \mu \mathrm{m}, \eta=2.4 \cdot 10^{3} \mathrm{~Pa} \cdot \mathrm{s}, \cos \vartheta \cong 1, \gamma \cong$ $20 \mathrm{mN} / \mathrm{m}$ ). The slow motion of the air-liquid interface in Fig. 4 could be due therefore to the presence of a capillary channel having a complex geometry.

Fig. 5 shows the detail of a contact region between two different yarns. After the inter-yarn pores have been filled, the liquid is seen to slowly creep in the relatively wide channel offered by the contact region. Because each contact region is shared by several inter-yarn pores, the liquid is transported from several inter-yarn pores simultaneously. Examination of other images show that the filling behavior illustrated in Fig. 5 occurs frequently.

The contact region is a relatively low-permeability path through which the liquid in the pores reaches the interior of the yarns. The side $(x z)$ view in Fig. 6 shows two yarns, one parallel and one perpendicular to the page. The top portion of the yarn parallel to the page is in contact with the droplet. Most of the yarn perpendicular to the page appears to be dry. For $t=25$ mins, the contact region is only partially filled with liquid. As time progresses, the contact region is progressively being replenished with liquid. From the contact region, the liquid is then transported into the interior of the yarns. Liquid is also transported directly from the drop to the interior of the yarns. The reduction in the extent of the dry regions within each yarn is particularly evident in the segmented images of Fig. 6 (right column).

Considered collectively, Figures 4,5 and 6 suggest a multi-stage transport mechanism (Fig. 7). In the first stage of liquid penetration, which we term pore penetration, the liquid is transported through the relatively large inter-yarn pores. During the pore penetration stage, some liquid also penetrates in the yarns that are directly in contact with the droplet. In the second stage (contact region filling) the liquid is transported mostly in the direction parallel to the plane of the textile through the channels corresponding to the regions of contact between the yarns. In the third stage (yarn saturation) the liquid slowly wicks towards the interior of the yarns flowing from the contact region and from the interior surfaces of the inter-yarn pores. In the case of the yarns that are in contact with the drop, the liquid originating from the contact region meets the liquid from the droplet until the yarn becomes fully saturated.

While the liquid distribution is three-dimensional, information about the volume-averaged saturation distribution is often useful. Theoretical analyses typically describe continuum volume-averaged fields. The theoretical model of Patnaik et al ${ }^{17}$, for example, assumes that transverse penetration occurs uniformly under the droplet, and that the liquid front is planar. In contrast, the model of Starov and coworkers ${ }^{28}$ assumes that the advancing liquid front in the material has a hemispherical shape with an increasing radius. Fig. 8 shows the shape of the region saturated with liquid as an $r-x$ curve. For any given value of $x, r$ is calculated as $r=$ $\sqrt{\mathrm{A}(\mathrm{x}) / \pi}$, where $\mathrm{A}(\mathrm{x})$ is the area of the wet region at $\mathrm{x}$. The shape reported in Fig. 8 is thus the high-saturation distribution averaged over the azimuthal angle. To calculate $\mathrm{A}(\mathrm{x})$ we applied trainable Weka Segmentation to every yz slice and computed the area of the liquid region.

The shape of the azimuthally-averaged high-saturation region in Fig. 8 resembles half an hour-glass. The highsaturation region below the drop is approximately hemispherical. The back of the textile is connected with the hemispherical region through a neck of initial radius $\cong$ $100 \mu \mathrm{m}$. As time proceeds, the radius of the neck increases, and the boundary of the hemispherical region shifts towards the back surface. In the region of minimal slope of the $x-r$ curve (for $r \cong 350 \mu \mathrm{m}$ ), the displacement of the boundary between $t=25$ mins and $\mathrm{t}=45$ mins is larger than between $\mathrm{t}=25$ mins and $\mathrm{t}=$ 45 mins, suggesting a slowing down of the average rate of liquid penetration. 
We further characterize the average motion of the liquid front by measuring absorption due to X-ray propagation in the yz plane, using the X-ray setup in radiography imaging mode. Fig. $9 A$ shows the $X$-ray intensity leaving the sample vs. the co-ordinate $x$ for selected times (the corresponding video is available in the Supporting Information). Assuming an inverse correlation between X-ray intensity and liquid saturation concentration, for any given value of $x$ the corresponding $\mathrm{X}$ ray intensity characterizes the area-averaged liquid saturation distribution in the corresponding yz plane. The high liquid saturation region within the textile corresponds to the range from $\mathrm{x} \cong 400 \mu \mathrm{m}$ to $700 \mu \mathrm{m}$ roughly. The range $150 \mu \mathrm{m}<\mathrm{x}<400 \mu \mathrm{m}$, approximately, corresponds to the portion of the drop outside the textile. The area-averaged position of the liquid-air interface corresponds to the region of largest intensity gradient near the back of the textiles, of which Fig. 9B provides an enlarged view.

We measure the motion of the high-gradient region corresponding to the liquid meniscus by calculating the shift in the $\mathrm{x}$ direction of the intensity profiles (see Materials and Methods section). The shift $\Delta \mathrm{x}_{\mathrm{L}}$ of the average $x$ co-ordinate of the liquid-air interface with respect to the front initial position is plotted in log-log scale against time for $\eta=97 \mathrm{~Pa} \cdot \mathrm{s}$ in Fig. $9 \mathrm{C}$, and for $\eta=$ $2.4 \cdot 10^{3} \mathrm{~Pa} \cdot \mathrm{s}$ in Fig. 9D, respectively. The data in Fig. 9C and 9D characterize the one-dimensional kinetics when the liquid-air interface is close to the back of the textile. Evaluation immediately following the deposition of the drop were inaccessible owing to the time needed to place the sample in the X-ray chamber and to reach a stable X-ray source intensity.

For both the smaller and larger viscosity liquids, a single power law does not fit the experimental data. For example, where $\eta=97 \mathrm{~Pa} \cdot \mathrm{s}$ the slope of the $\Delta \mathrm{x}_{\mathrm{L}}-\mathrm{t}$ curve is smaller than 0.5 (the value predicted by the Lucas-Washburn law) for $t>1000$ s roughly. Such a poor fit to the data qualitatively highlights the limitation of the single power law description. An observable variation in the liquid penetration behavior shown in Fig. 9 might be due to differences in observation window. Assuming that the penetration rate is inversely proportional to the viscosity, the features corresponding to $t=$ 1000 s in Fig. 9C would correspond to $t=24700$ s in Fig. 9D, while our observation window for $\eta=2.4$. $10^{3} \mathrm{~Pa} \cdot \mathrm{s}$ extends only up to $\mathrm{t}=6000 \mathrm{~s}$.

A single power-law would be expected if: i) entrance effects associated with the small length of the capillary channels did not alter the dynamics of the liquid penetration process and ii) the liquid transport was governed by a single transport law for any value of $t$. Continuum theories for liquid transport in porous media, such as the Lucas-Washburn theory ${ }^{16}$ or variations of it $^{32,33}$, are derived under the assumption that the porous medium can be modelled as a collection of paral- lel channels having length $\mathrm{L} \gg \mathrm{R}$, where $\mathrm{R}$ is the average pore radius ${ }^{16}$. By contrast, in our case, even the largest pores that control the initial motion of the liquid have an aspect ratio close to 1 .

Staples and Shaffer ${ }^{34}$ examined the law of propagation of a liquid meniscus in a capillary tube of sinusoidal cross section. They proved that if the length of the capillary tube is much larger than the wavelength of the sinusoid, the predicted liquid meniscus position fluctuates around the curve predicted by the Lucas-Washburn law. This behavior can be understood by considering the speed of propagation $\frac{d L}{d t}$ of a meniscus in a circular channel of non-uniform cross sectional radius $\mathrm{R}(\mathrm{z})^{34}$ :

$$
\frac{\mathrm{dL}}{\mathrm{dt}}=\frac{1}{4} \frac{\gamma}{\eta} \frac{\cos \theta}{[\mathrm{R}(\mathrm{L})]^{3}} \frac{1}{\mathrm{I}(\mathrm{L})} \quad \text { Eq. (1) }
$$

where

$$
\mathrm{I}(\mathrm{L})=\int_{0}^{\mathrm{L}} \frac{\mathrm{dz}}{[\mathrm{R}(\mathrm{z})]^{4}} \quad \text { Eq. (2) }
$$

For $\mathrm{R}=$ const., integration of Eq. (1) gives $\mathrm{L}^{2}=$ $\frac{1}{2} \frac{\gamma}{\eta} \cos \theta$ Rt, i.e., Lucas-Washburn's law. For a small sinusoidal perturbation to a constant radius channel, $\mathrm{R}=$ $\mathrm{R}_{0}[1-\varepsilon \sin (\lambda \mathrm{z})]$ with $\varepsilon \ll 1$. In this case, $\mathrm{I} \simeq \frac{\mathrm{L}}{\mathrm{R}_{0}^{4}}+$ $4 \frac{\varepsilon}{\mathrm{R}_{0}^{4}} \int_{0}^{\mathrm{L}} \sin \lambda \mathrm{zdz}$. For $\mathrm{L} \gg \lambda$, the integral of the sine function has zero average, and the Lucas-Washburn law is recovered in a time-averaged sense. However, for $\lambda \sim \mathrm{L}$, the solution of Eq. (1) gives a marked non-linear variation from the Lucas-Washburn law, whose features depend on the actual geometry of the pore ${ }^{34}$. This simple model illustrates that applying results for thick multilayer textiles to single-layer textiles can give qualitatively and quantitatively incorrect results, and could explain the non-linear behavior seen in Fig. 9C and 9D.

An important aspect that we believe could explain the slow down observed in Fig. 9C and 9D is the change in dominant direction of liquid propagation as time progresses. Using the terminology introduced in the discussion of Fig. 7, in the pore filling stage the liquid is transported mainly in the x direction. However, in the contact region filling and yarn saturation stages the fluid is mostly transported in the lateral (in-plane) direction, contributing little to the $\mathrm{x}$ component of the average fluid velocity. 


\section{CONCLUSIONS}

We have examined by X-ray tomography and radiography imaging the time-dependent transport of ultrahigh viscosity liquids in the transplanar (normal) direction following the deposition of a small droplet on the surface of a single-layer textile.

A key insight of our investigation is that the transverse liquid transport appears to proceed in stages, each stage being associated to a geometric feature of the fiber arrangement. The fast initial transport appears to be mainly associated to the fluid filling the large, interyarn pores. The late stages of penetration are associated to the slow impregnation of the interior of the yarns. In the intermediate stage where the liquid flows mostly through the region of contact between the yarns.

We have quantified the one dimensional kinetics of liquid penetration by using $X$-ray radiography. The results show that a single power law does not capture all the data. The asymptotic transport rate is much smaller than that predicted by the Lucas-Washburn equation.

Models that consider single layer textiles as homogeneous and isotropic porous media have been applied to lateral, in plane transport with some success ${ }^{9,35}$. This success is likely due to the fact that in the lateral direction the asymptotic flow is to a first approximation independent of the microscopic geometry of the textile. In our case, the small thickness of the single layer textile can prevent an asymptotic flow behavior being established before the liquid reaches the back surface of the textile. This feature could explain the strong deviations from a power law behavior seen in Fig. $9 \mathrm{C}$ and 9D.
In addition, to aid the development of theoretical models, our results may have implications for the development of strategies to manipulate liquid transport in textiles. For example, the XCT images suggest that the initial liquid mass flux is governed by transport in the relatively large inter-yarn pores. Altering these features, for instance by utilizing nanoparticles to partially obstruct the pores, could represent an approach to tailor the liquid transport rates in the transplanar direction.

\section{ASSOCIATED CONTENT}

\section{Supporting information}

Radiography video of transverse transport of a droplet for $\eta=97 \mathrm{~Pa} \cdot \mathrm{s}$. This supporting information is available free of charge on the ACS Publication Website at DOI:

\section{AUTHOR INFORMATION}

\section{Corresponding Author}

* Professor Asa H. Barber. Email: asa.barber@port.ac.uk Tel: +44 (0)2392842363

\section{Notes}

The authors declare no competing financial interest

\section{ACKNOWLEDGMENT}

This research is generously supported by the Material Research Institute (MRI) of Queen Mary university of London and the Defense Science \& Technology Laboratory (DSTL). The authors thank School of Engineering in University of Portsmouth for granting access to the Zeiss 520 Versa X-ray microscope and Dr. Alexander Kao for his assistance in the experiments.

\section{REFERENCES}

1. Brewer, S. A., \& Willis, C. R. Structure and oil repellency: textiles with liquid repellency to hexane. Appl. Surf. Sci. 2008, 254 (20), 6450-6454.

2. Leng, B., Shao, Z., de With, G., \& Ming, W. . Superoleophobic cotton textiles. Langmuir 2009, 25 (4), 24562460.

3. Advani, S. G., Bruschke, M. V., \& Parnas, R. S. . Resin transfer molding flow phenomena in polymeric composites. Compos. Mater. Ser. 1994, 465-465.

4. $\quad$ Abrate, S. Resin flow in fiber preforms. Appl. Mech. Rev. 2002, 55 (6), 579-599.

5. Birrfelder, P., Dorrestijn, M., Roth, C., \& Rossi, R. M. . Effect of fiber count and knit structure on intra-and inter-yarn transport of liquid water. Text. Res. J. 2013, 83 (14), 1477-1488.

6. $\quad$ Bartels, V. T. Physiological comfort of sportswear. Textiles in sport 2005, 177-203.

7. Pezron, I., Bourgain, G., \& Quéré, D. Imbibition of a fabric. J. Colloid Interface Sci. 1995, 173 (2), 319-327.

8. Adler, M. M., \& Walsh, W. K. . Mechanisms of transient moisture transport between fabrics. Text. Res. J. 1984, 54 (5), 334-343.

9. Kissa, E. Capillary sorption in fibrous assemblies. J. Colloid Interface Sci. 1981, 83 (1), 265-272.

10. Kissa, E. Wetting and wicking. Text. Res. J. 1996, 66 (10), 660-668. 
11. Miller, B., \& Tyomkin, I. Spontaneous transplanar uptake of liquids by fabrics. Text. Res. J. 1984, 54 (11), 706-712.

12. Sarkar, M., Fan, J., \& Qian, X. Transplanar water transport tester for fabrics. Meas. Sci. Technol. 2007, 18 (5), 1465.

13. Tang, K. P. M., Kan, C. W., \& Fan, J. T. . Evaluation of water absorption and transport property of fabrics. Text. Prog. 2014, 46 (1), 1-132.

14. Gillespie, T. The spreading of low vapor pressure liquids in paper. J. Colloid Sci. 1958, 13 (1), 32-50.

15. Adams, K. L., \& Rebenfeld, L. In-plane flow of fluids in fabrics: structure/flow characterization. Text. Res. J. 1987, 57 (11), 647-654.

16. Washburn, E. W. The dynamics of capillary flow. Phys. Rev. 1921, 17 (3), 273.

17. Patnaik, A., Rengasamy, R. S., Kothari, V. K., \& Ghosh, A. Wetting and wicking in fibrous materials. Text. Prog. 2006, 38 (1), 1-105.

18. Kawase, T., Sekoguchi, S., Fuj, T., \& Minagawa, M. Spreading of liquids in textile assemblies: Part I: Capillary spreading of liquids. Text. Res. J. 1986, 56 (7), 409-414.

19. Perwuelz, A., Mondon, P., \& Caze, C. . Experimental study of capillary flow in yarns. Text. Res. J. 2000, 70 (4), 333-339.

20. Danino, D., \& Marmur, A. Radial capillary penetration into paper: limited and unlimited liquid reservoirs. J. Colloid Interface Sci. 1994, 166 (1), 245-250.

21. Salminen, P. Studies of water transport in paper during short contact times. PhD, Åbo Akademi1988.

22. Bencsik, M., Adriaensen, H., Brewer, S. A., \& McHale, G. . Quantitative NMR monitoring of liquid ingress into repellent heterogeneous layered fabrics. J. Magn. Reson. 2008, 193 (1), 32-36.

23. Rossi, R. M., Stämpfli, R., Psikuta, A., Rechsteiner, I., \& Brühwiler, P. A. Transplanar and in-plane wicking effects in sock materials under pressure. Text. Res. J. 2011, 0040517511413317.

24. Weder, M., Brühwiler, P. A., \& Laib, A. . X-ray tomography measurements of the moisture distribution in multilayered clothing systems. Text. Res. J. 2006, 76 (1), 18-26.

25. Bayramli, E., \& Powell, R. L. . The normal (transverse) impregnation of liquids into axially oriented fiber bundles. J. Colloid Interface Sci. 1990, 138 (2), 346-353.

26. Karppinen, T., Kassamakov, I., Aaltonen, J., Pajari, H., \& Hæggström, E. . Measuring liquid penetration in the thickness direction of paper. The European Physical Journal Applied Physics 2005, 32 (1), 65-71.

27. Hyväluoma, J., Raiskinmäki, P., Jäsberg, A., Koponen, A., Kataja, M., \& Timonen, J. Simulation of liquid penetration in paper. Phys. Rev. E 2006, 73 (3), 036705.

28. Starov, V. M., Zhdanov, S. A., \& Velarde, M. G. Spreading of liquid drops over thick porous layers: complete wetting case. Langmuir 2002, 18 (25), 9744-9750.

29. Rudolf, M., Boutelier, D., Rosenau, M., Schreurs, G., \& Oncken, O. Rheological benchmark of silicone oils used for analog modeling of short-and long-term lithospheric deformation. Tectonophysics 2016, 684, $12-22$.

30. Arganda-Carreras I, K. V., Rueden C, Eliceiri KW, Schindelin J, Cardona A, Seung HS. Trainable Weka Segmentation: a machine learning tool for microscopy pixel classification. Bioinformatics 2017.

31. Courbin, L., Denieul, E., Dressaire, E., Roper, M., Ajdari, A., \& Stone, H. A. . Imbibition by polygonal spreading on microdecorated surfaces. Nat. Mater. 2007, 6 (9), 661-664.

32. Marmur, A. The radial capillary. J. Colloid Interface Sci. 1988, 124 (1), 301-308.

33. Marmur, A., \& Cohen, R. D. Characterization of porous media by the kinetics of liquid penetration: the vertical capillaries model. J. Colloid Interface Sci. 1997, 189 (2), 299-304.

34. Staples, T. L., \& Shaffer, D. G. Wicking flow in irregular capillaries. Colloids and Surfaces A: Physicochemical and Engineering Aspects 2002, 204 (1), 239-250.

35. Marmur, A. Drop penetration into a thin porous medium. J. Colloid Interface Sci. 1988, 123 (1), 161-169. 


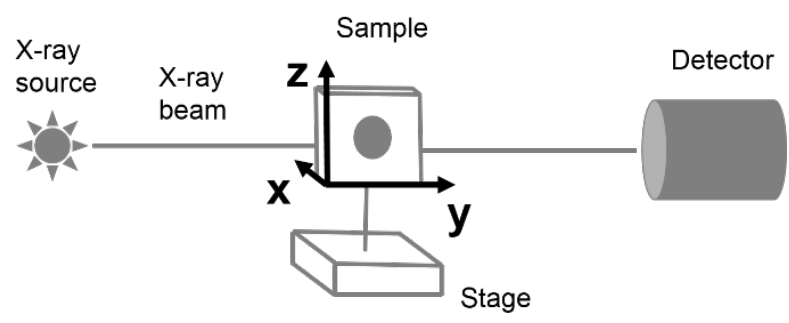

Figure 1. Schematic of the experimental setup. The dark circle in the sample represents the base of the droplet (plane $x=0$ ). The interior of the textile is in the positive $x$ direction.

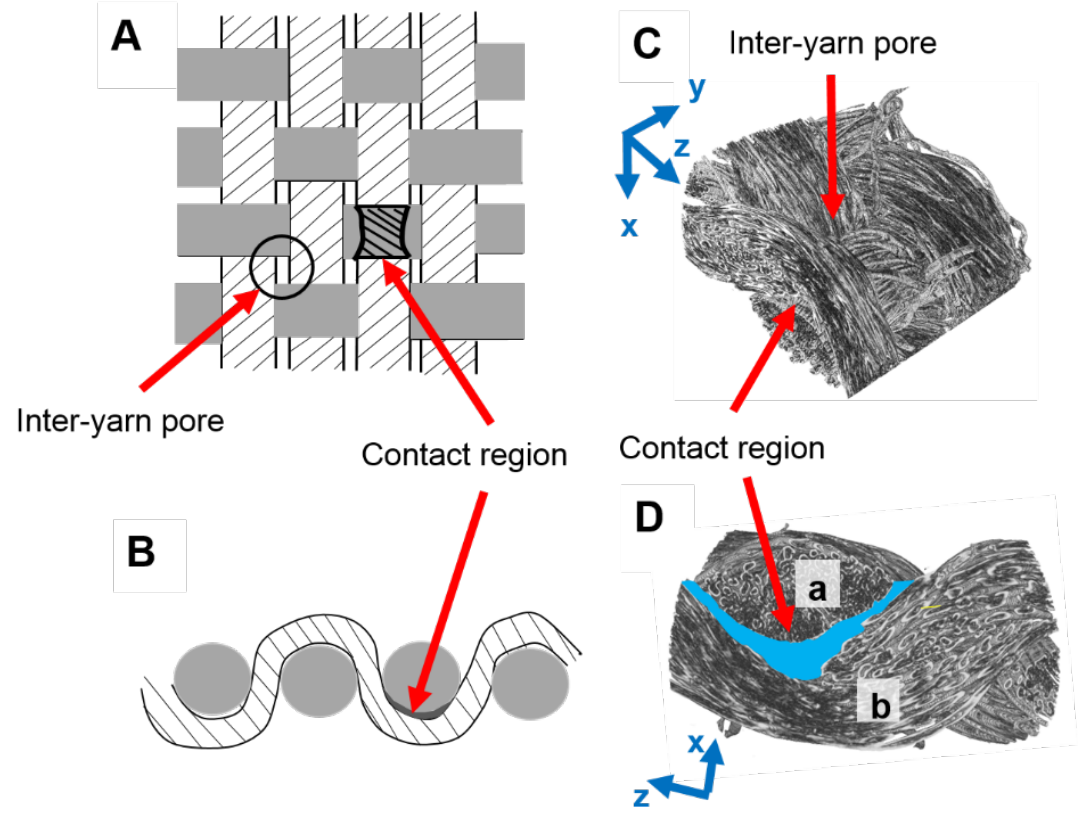

Figure 2. Schematics $(A, B)$ and XCT images $(C, D)$ illustrating the structure of the textile.

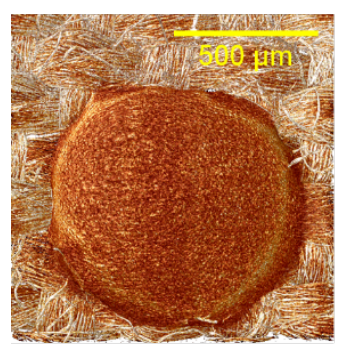

$y z$ view (front)
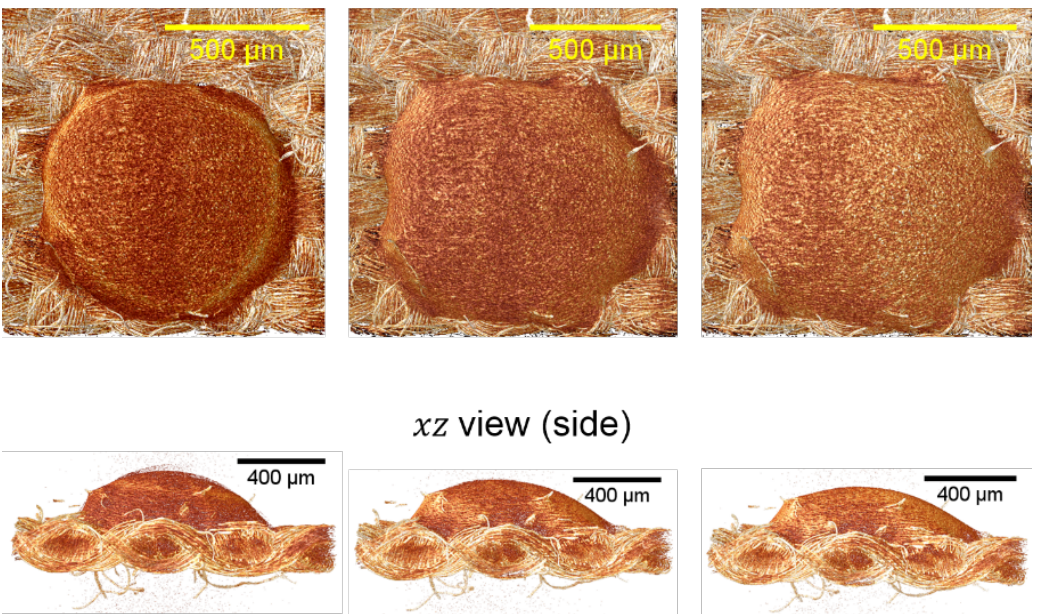

Figure 3. Tomography images showing the shape of the drop in the outside of the textile (online version in color). From left to right: $t=25 \mathrm{mins}, \mathrm{t}=45 \mathrm{mins}$ and $\mathrm{t}=70 \mathrm{mins}$, respectively. The liquid viscosity is $\eta=2.4 \cdot 10^{3} \mathrm{~Pa} \cdot \mathrm{s}$. 

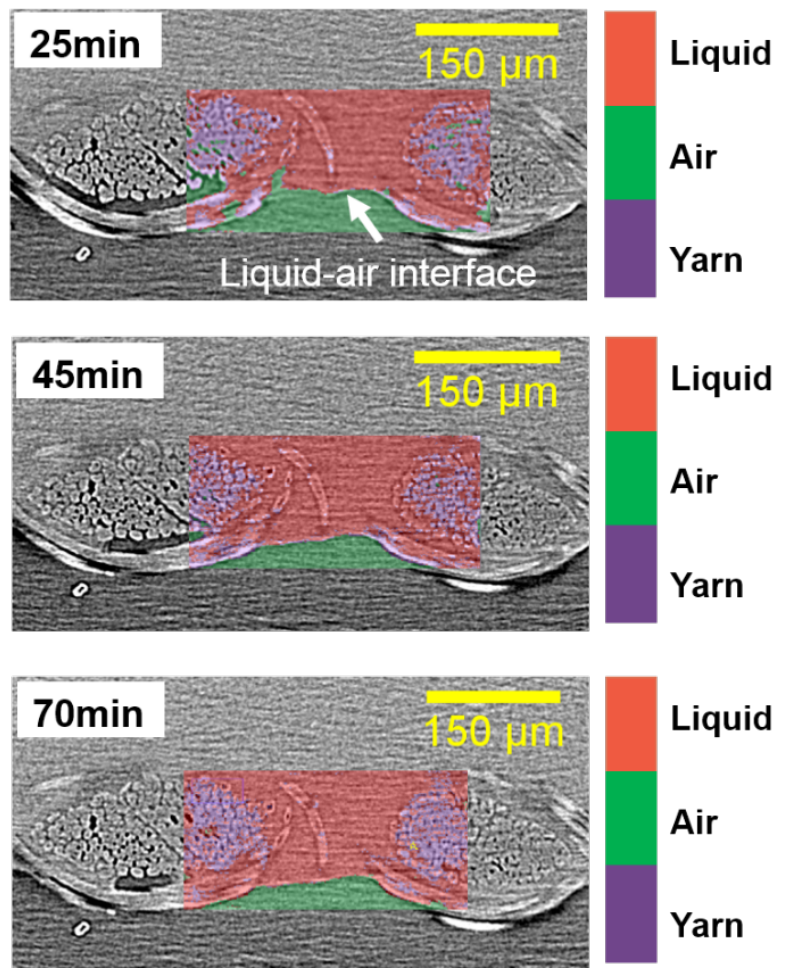

Figure 4. $x z$ slice of liquid distribution in an inter-yarn pore at successive times for $\eta=2.4 \cdot 10^{3} \mathrm{~Pa} \cdot \mathrm{s}$. (Online version in color)
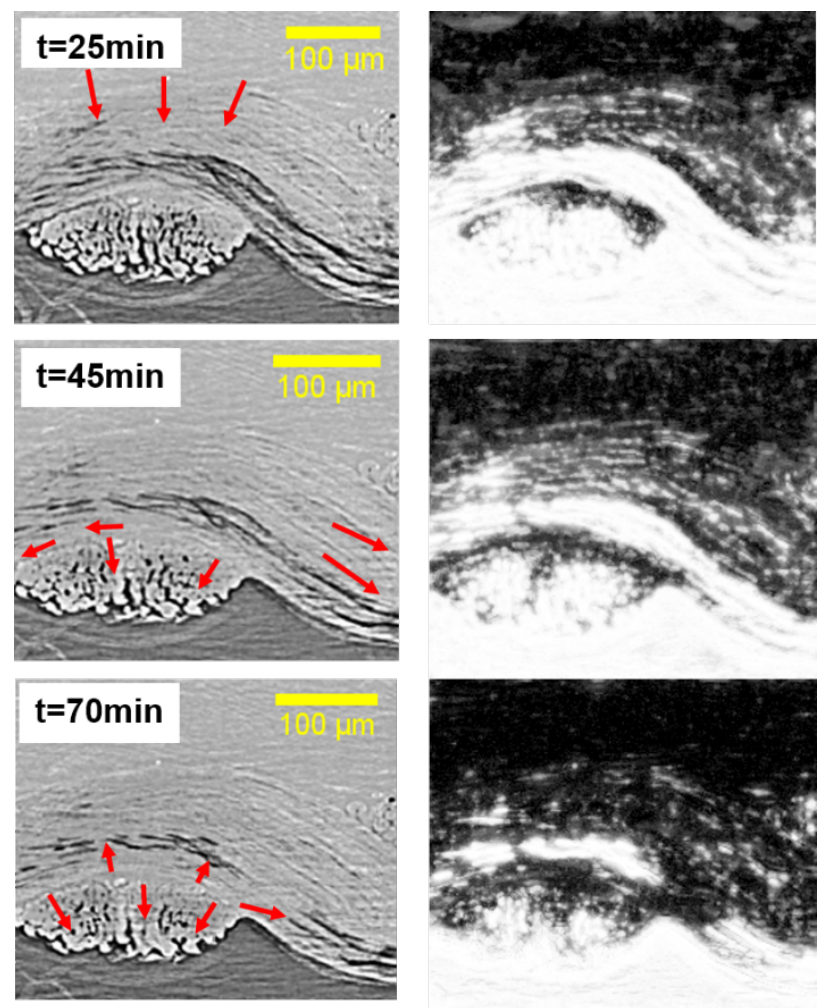

Figure 6. Left: $x z$ slice of liquid distribution in the neighborhood of the contact region between yarns for $\eta=2.4$. $10^{3} \mathrm{~Pa} \cdot \mathrm{s}$. Arrows indicate the approximate direction of the liquid flow. Right: corresponding segmented images, showing the liquid in black.
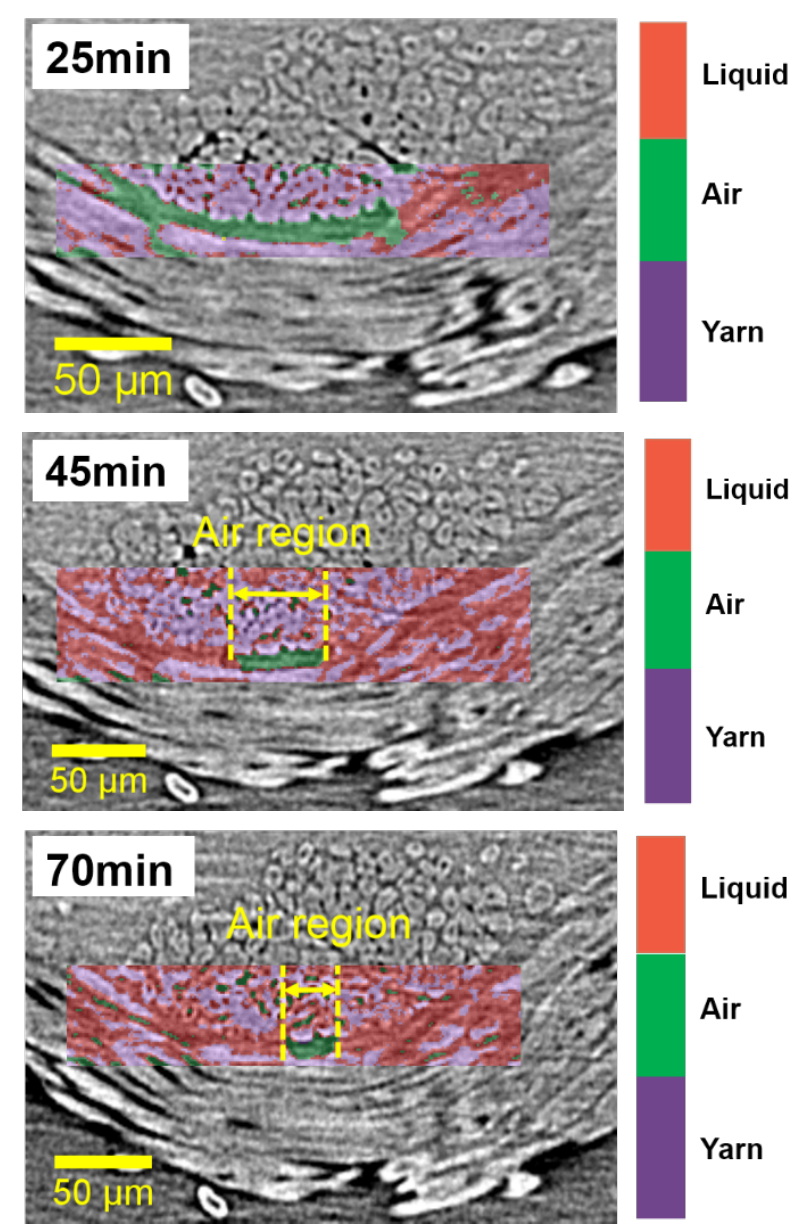

Figure 5. $x z$ slice showing the progressive filling of the contact region by the liquid for $\eta=2.4 \cdot 10^{3} \mathrm{~Pa} \cdot s$.. (Online version in color).

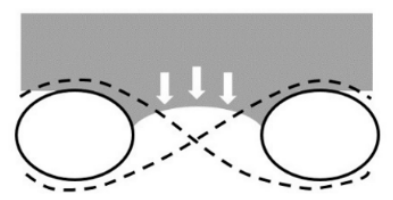

Pore penetration

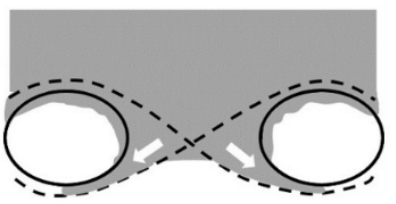

Contact region filling

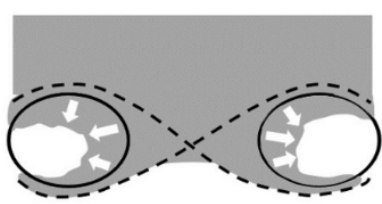

Yarn saturation

Figure 7. Three stages of transplanar penetration suggested by the XCT images. 


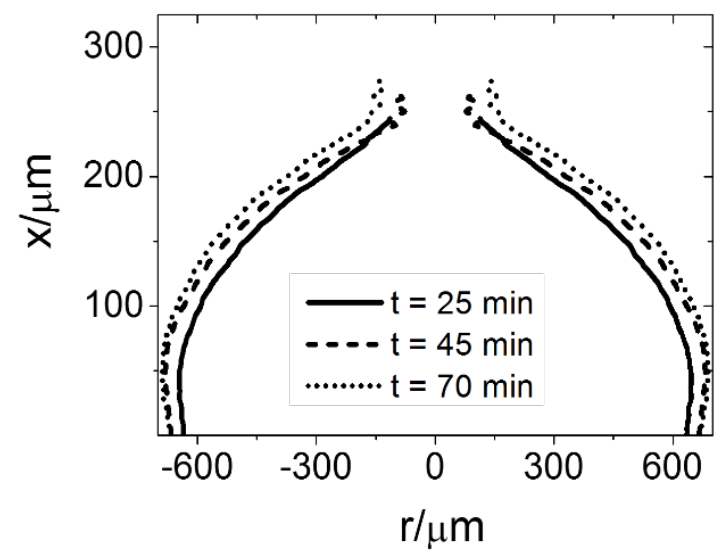

Figure 8. Radius of wetted region inside the textile vs $x$ coordinates for $\eta=2.4 \cdot 10^{3} \mathrm{~Pa} \cdot s$.
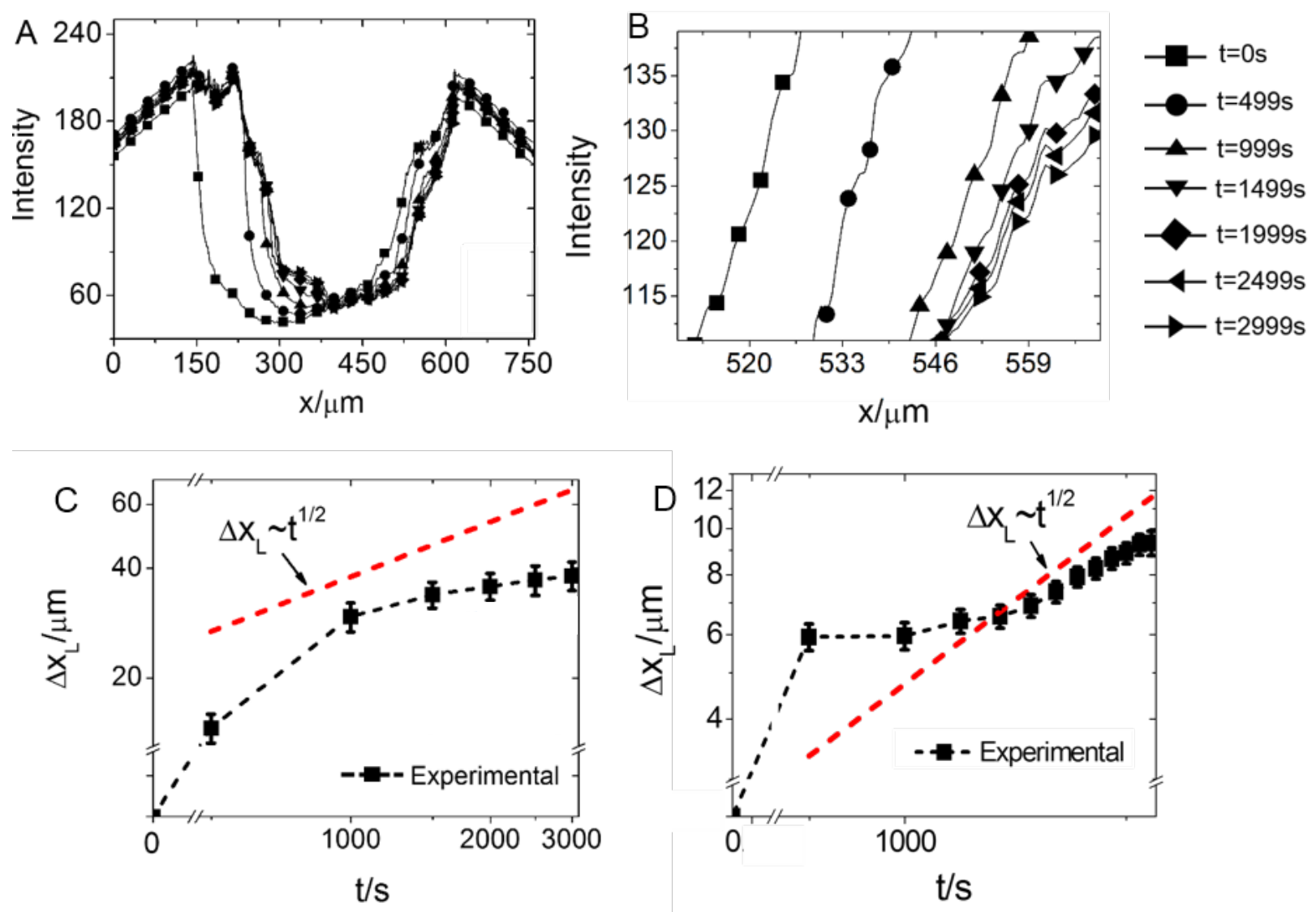

Figure 9. A: profile of X-ray intensity vs $x$ coordinates for successive times and $\eta=97 \mathrm{~Pa} \cdot s$. B: zoom in the region $x \in[511,567]$. C: log-log plot of the average displacement of the liquid front as a function of time for $\eta=97 P a \cdot s$. D: the same as $\mathrm{C}$ for $\eta=2.4 \cdot 10^{3} \mathrm{~Pa} \cdot \mathrm{s}$. 

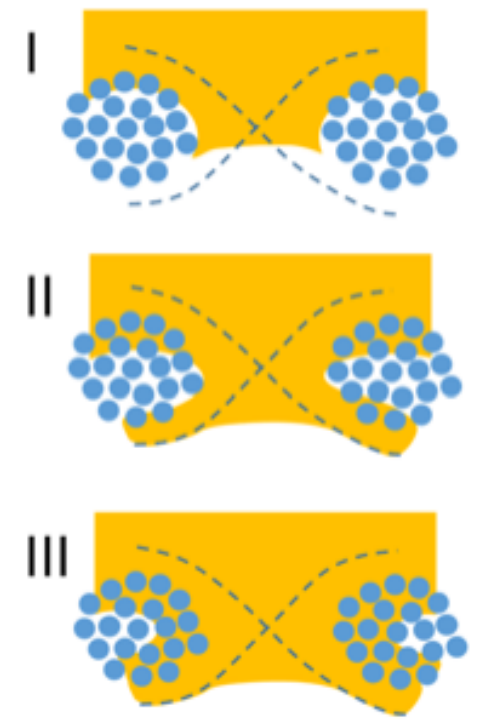
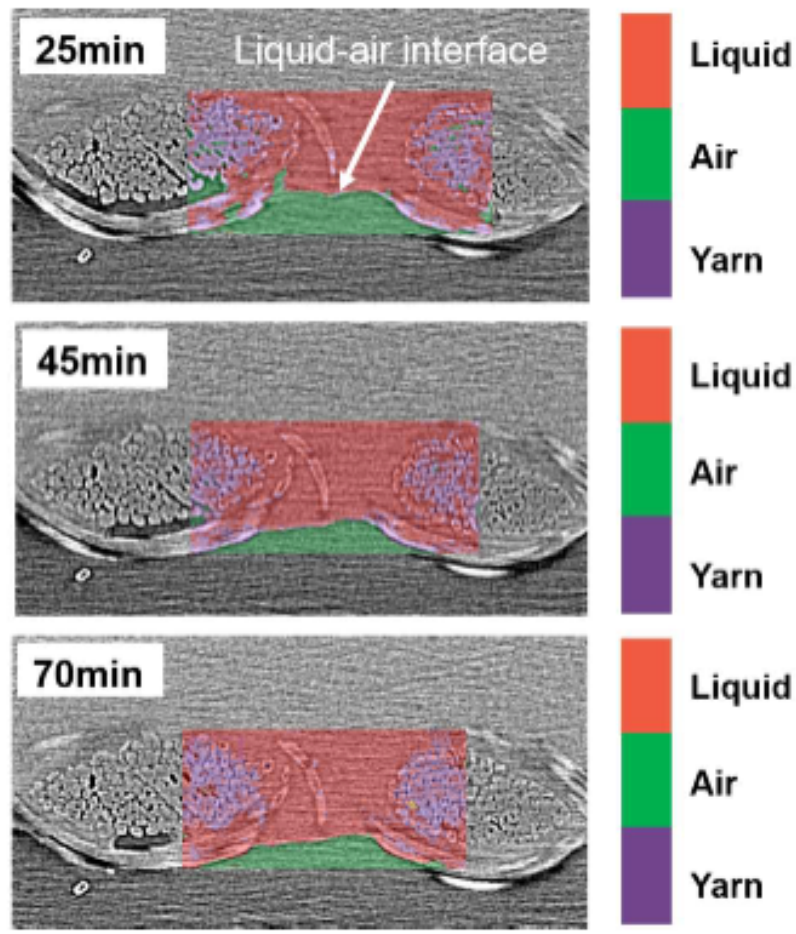